\title{
Examining the Relation Between the Performance Levels of the Footballers and the Data Obtained With the Routine "Combined Performance Analyses"*
}

\author{
Bülent TURNA \\ Celal Bayar University, \\ Manisa, Turkey
}

\author{
Fatih KILINÇ \\ Süleyman Demirel University, \\ Isparta, Turkey
}

\begin{abstract}
The purpose of the study is to investigate the relationship between game performance levels depending on the combined performance analysis conducted routinely on footballers. Eighteen players from Tekirova Belediyespor, which is a third league football team in Turkey, participated in the study. The average ages, heights, and weights of the players, who voluntarily took part in the study, were $24.28 \pm 2.63$ years, $180 \pm 6.48$ centimeters $(\mathrm{cm})$, and 77.78 \pm 7.31 kilograms $(\mathrm{kg})$, respectively. In the study, right and left hand grip strength, Subcutaneous fat measurement at eight different spots, back strength, leg strength, single right leg vertical jump, single left leg vertical jump, double leg free vertical jump, 30-meter speed test, flexibility tests, heartbeats at rest, systolic and diastolic blood-pressures were measured. The body fat percentage was calculated through the Lange formula. Besides, the anaerobic power values were calculated through the Lewis formula. In addition to this, the games Tekirova Belediyespor versus (vs.) Sandıklı Spor in the 20th week, Tekirova Belediyespor vs. Bergamaspor in the 26th week, Tekirova Belediyespor vs. Üsküdar Anadoluspor in the 33rd week were analyzed through the tally method. As for the statistical procedures, the Statistical Package for Social Sciences (SPSS) 18 multifactor analysis of variance (ANOVA) test was used for the comparison of the values of different tests, and the SPSS 18 Pearson one-way correlation test was used to examine the relationship between game analyses and combined performance analyses. In the study, a statistically significant difference could not be determined between the values of the combined performance analysis values and actual game performance levels $(p>0.05)$.
\end{abstract}

Keywords: combined analysis, football, biomotoric properties

\section{Introduction}

Performance is the score that an athlete can exert physically, physiologically, biomotorically, psycho-mentally, technically, and tactically (Kılınç, 2008). An extraordinary development has been seen in performance-based sports recently. Many levels that seemed difficult to reach in the past have been reached and performance limits have been improved extraordinarily. Well-planned training methods aimed at improving physiological, technical-tactical, and conditional qualities in order to raise athletic efficiency in football.

\footnotetext{
* This work was summarized in the Master's degree in Health Sciences Institute of Süleyman Demirel University. Bülent TURNA, Ph.D. candidate, student, Institute of Health Sciences, Celal Bayar University. Fatih KILINÇ, Ph.D., professor, Faculty of Sport Sciences, Süleyman Demirel University.
} 
Although the game without a ball has an important place in football, it is those movements made with the ball that determine the result. Today, methods based on positive sciences must be followed in order to achieve success in football as in all other sport types. Anthrometric and physiological studies contribute to the athlete' chose of a suitable type for training and foresight for the aimed success (Duyul, 2005). Biomotoric qualities are regarded as the basic movement skills of a human being. These are endurance, strength, speed, dynamism, flexibility, and coordination (Duyul, 2005). Game analysis is a frequently used method in order to determine the performance levels of the players and the teams. Analysis programs enable us to collect all the data related to the movements in a game and have access to the required information instantly. In addition, they make it possible to analyze and assess the physical, technical, and tactical performance of the player in the game. The results obtained from the analyses help trainers to determine the type of trainings by providing information about the efficiency of the team and the footballers. Trainers benefit from note-taking analysis system in order to base their decisions on game analysis data and collect as much information as possible. When performance analysis is discussed, trainers must take into account how their analyses comply with the "training-game cycle." In addition, performance analysis can be adjusted according to a yearly plan, such as pre-season, mid-season, and post-season. Therefore, it is possible to perform a situation assessment at every stage (Carling, Williams, \& Reily, 2007).

\section{The Players Who Participated in the Study}

Eighteen professional players from Tekirova Belediyespor, a third league football team in Turkey, participated in the study. The average age of the players, who voluntarily participated in the research was 24.28 \pm 2.63 years, average height was $180 \pm 6.48$ centimeters $(\mathrm{cm})$, and average weight was $77.78 \pm 7.31$ kilograms $(\mathrm{kg})$. All the tests were conducted in the field where the game would be played. The tests began at $10 \mathrm{o}$ 'clock in the morning. All the participating players were made to do warmup, mobility, flexibility and coordination, rhythm and balance exercises based on warmup activities in a systematic manner. A minimum of two trials were done for each test in order to determine the optimum value.

\section{Anthropometric Measurements}

The height measurement was conducted in the right anatomical posture with the feet naked and adjacent. The weight measurement was done with the players in underwear and feet naked. A weighing machine with $0.1 \mathrm{~kg}$ precision was used for the measurements. A Holtain brand skinfold caliper was employed in order to conduct the subcutaneous fat measurements. The measurements were conducted at eight points: biceps, triceps, subscapula, pectoral, abdominal, iliac, quadriceps, and calf. The Lange formula was used in order to determine the body fat percentage of the players.

$$
\text { Lange }=(\text { biceps }+ \text { triceps }+ \text { subscapula }+ \text { pectoral }+ \text { iliac }+ \text { quadriceps }) \times 0.097+3.64
$$

\section{Physiological Tests}

The resting heartbeat rates and the systolic and diastolic blood pressures of the players were measured one hour after the breakfast. The measurement was conducted at the left wrist in sitting posture with a Bosch brand electronic sphygmomanometer. After the anaerobic power data, free jump data and body weight data were taken, and the anaerobic power was found by using the Lewis formula.

$$
P=\sqrt{ } 4.9 \times(\text { weight }) \times \sqrt{ } \text { Free vertical jump data }
$$




\section{Biomotoric Tests}

\section{Free Vertical, Right Foot Single, and Left Foot Single Jumping Tests}

Takei brand jump meter was tied to the abdominal area, and the players were asked to jump vertically with both feet and arms free. This was conducted in fixed spot. After two trials, the highest value was recorded.

\section{Right-Left Hand Grip Force Tests}

A Takei brand dynamometer was attached to the abdomen area of the players, and they were asked to jump vertically in a hands-free position, and then land within a certain area. After two trials, the highest value was recorded as centimeters.

\section{Thirty-Meter Sprint Test}

A Technique brand photocell and programmed computer system were used. Firstly, the player started at 50 $\mathrm{cm}$ off the front part of the start photocells, and then, ran through an intermediary spot in between and arrived at the finish point. The scores obtained were recorded as split seconds.

\section{Back Force Test}

A power dynamometer (back/leg chest dynamometer, Takei, Japan) was used. The player was upright with the arms folded 90-degree at the elbows. A force was applied with 4-minute intervals and the best score of the three trials was recorded.

\section{Leg Force Test}

A Takei brand dynamometer was used for this test. A force was applied while the body was slightly tilted forward, the arms stretched, and the legs bent 120 degrees. Three trials with 4-minute intervals were performed and the best score was recorded (Keyserling, Herrin, \& Chaffin, 1978).

\section{Flexibility (Sit and Reach) Test}

The player sat on the flexibility stand, which complied with the Eurofit test battery. After two trials, the highest score was recorded as $\mathrm{cm}$.

\section{Game Analysis}

Hand marking, which is considered as one of the most widespread systems, was employed. First, each game was watched live. After that, the recorded game was watched and analyzed on a computer. In order to reduce the margin of error to the minimum, many movements determined previously were arranged as in the table and the move performed was recorded in the column in the table by using hand marking. By taking into account the risk of missing the shirt number of a player, we worked on the game system, and the game was watched a few times prior to the analysis. At the end of the game, the number of each successful or unsuccessful movement was multiplied by two and the negative score was subtracted from the positive score, which gave us the performance score.

\section{Statistics}

Eighteen professional footballers from Tekirova Belediyespor, a third league football team in Turkey, participated in the study. The games in the 20th, 26th, and 33rd weeks, which were hosted by them, were studied. The measurements of the players, who were chosen for the first 11 and who were likely to be included in the game later, were taken one day before the game, and the Statistical Package for Social Sciences (SPSS) 
18 analysis of variance (ANOVA) test was used in order to study the differences between the weeks. The SPSS 18 Pearson one-way correlation test was employed in order to study the relation between the data and the analysis of the game, which was made with the tally score method by using the video recordings of the game.

\section{Results and Discussion}

Table 1

Physical Properties of the Tekirova Belediyespor Football Team

\begin{tabular}{lcccc}
\hline & $N$ & Minimum & Maximum & Art. Ave. \pm S.D. \\
\hline Age & & 20 & 30 & $24.28 \pm 2.63$ \\
Height $(\mathrm{cm})$ & 14 & 172 & 193 & $180 \pm 6.48$ \\
Weight $(\mathrm{kg})$ & & 66 & 92 & $77.78 \pm 7.31$ \\
\hline
\end{tabular}

Table 2

Comparison of the Subcutaneous Fat Measurement Values of the Tekirova Belediyespor Football Team Between the Tests

\begin{tabular}{|c|c|c|c|c|c|c|c|}
\hline & Tests & $N$ & Minimum & Maximum & Art. Ave. \pm S.D. & $D f$ & $p$ \\
\hline \multirow{4}{*}{$\begin{array}{l}\text { Biceps } \\
\text { (mmhg.) }\end{array}$} & 1. Test & & 1.00 & 9.00 & $3.58 \pm 2.2$ & & \multirow{4}{*}{0.905} \\
\hline & 2. Test & 14 & 2.00 & 9.40 & $3.82 \pm 2.2$ & & \\
\hline & 3. Test & & 1.20 & 9.40 & $3.81 \pm 2.2$ & & \\
\hline & Total & 42 & 1.00 & 9.40 & $3.74 \pm 2.1$ & & \\
\hline \multirow{4}{*}{$\begin{array}{l}\text { Triceps } \\
\text { (mmhg.) }\end{array}$} & 1. Test & & 4.20 & 17.40 & $9.97 \pm 4.3$ & & \multirow{4}{*}{0.964} \\
\hline & 2. Test & 14 & 4.30 & 17.60 & $10.10 \pm 4.3$ & & \\
\hline & 3. Test & & 4.00 & 15.20 & $9.68 \pm 3.9$ & & \\
\hline & Total & 42 & 4.00 & 17.60 & $9.92 \pm 4.1$ & & \\
\hline \multirow{4}{*}{$\begin{array}{l}\text { Subscapular } \\
\text { (mmhg.) }\end{array}$} & 1. Test & & 7.00 & 14.00 & $9.35 \pm 2.6$ & & \multirow{4}{*}{0.979} \\
\hline & 2. Test & 14 & 7.00 & 14.60 & $9.51 \pm 2.6$ & & \\
\hline & 3. Test & & 7.00 & 14.00 & $9.31 \pm 2.6$ & & \\
\hline & Total & 42 & 7.00 & 14.60 & $9.39 \pm 2.6$ & & \\
\hline \multirow{4}{*}{$\begin{array}{l}\text { Pectoral } \\
\text { (mmhg.) }\end{array}$} & 1. Test & & 4.00 & 13.00 & $8.15 \pm 3.4$ & & \multirow{4}{*}{0.965} \\
\hline & 2. Test & 14 & 4.20 & 13.20 & $8.44 \pm 3.4$ & & \\
\hline & 3. Test & & 3.80 & 12.60 & $8.12 \pm 3.4$ & & \\
\hline & Total & 42 & 3.80 & 13.20 & $8.24 \pm 3.3$ & $\begin{array}{r}2 \\
30\end{array}$ & \\
\hline \multirow{4}{*}{$\begin{array}{l}\text { Abdominal } \\
\text { (mmhg.) }\end{array}$} & 1. Test & & 6.00 & 23.00 & $14.25 \pm 5.7$ & $\begin{array}{l}39 \\
41\end{array}$ & \multirow{4}{*}{0.968} \\
\hline & 2. Test & 14 & 6.20 & 23.60 & $14.67 \pm 5.9$ & & \\
\hline & 3. Test & & 6.00 & 22.80 & $14.12 \pm 5.8$ & & \\
\hline & Total & 42 & 6.00 & 23.60 & $14.35 \pm 5.7$ & & \\
\hline \multirow{4}{*}{$\begin{array}{l}\text { Iliac } \\
\text { (mmhg.) }\end{array}$} & 1. Test & & 2.40 & 10.00 & $6.08 \pm 2.7$ & & \multirow{4}{*}{0.981} \\
\hline & 2. Test & 14 & 2.40 & 10.40 & $6.20 \pm 2.8$ & & \\
\hline & 3. Test & & 2.20 & 9.80 & $5.99 \pm 2.7$ & & \\
\hline & Total & 42 & 2.20 & 10.40 & $6.09 \pm 2.7$ & & \\
\hline \multirow{4}{*}{$\begin{array}{l}\text { Quadriceps } \\
\text { (mmhg.) }\end{array}$} & 1. Test & & 6.20 & 16.00 & $11.38 \pm 3.3$ & & \multirow{4}{*}{0.987} \\
\hline & 2. Test & 14 & 6.40 & 16.40 & $11.44 \pm 0.3$ & & \\
\hline & 3. Test & & 6.20 & 16.40 & $11.24 \pm 3.3$ & & \\
\hline & Total & 42 & 6.20 & 16.40 & $11.35 \pm 3.2$ & & \\
\hline \multirow{4}{*}{$\begin{array}{l}\text { Calf } \\
\text { (mmhg.) }\end{array}$} & 1. Test & & 5.40 & 22.00 & $10.32 \pm 4.7$ & & \multirow{4}{*}{0.992} \\
\hline & 2. Test & 14 & 5.00 & 21.20 & $10.21 \pm 4.6$ & & \\
\hline & 3. Test & & 5.20 & 21.00 & $10.10 \pm 4.5$ & & \\
\hline & Total & 42 & 5.00 & 22.00 & $10.21 \pm 4.5$ & & \\
\hline
\end{tabular}


(Table 2 to be continued)

\begin{tabular}{|c|c|c|c|c|c|c|}
\hline \multirow{4}{*}{$\begin{array}{l}\text { Body fat } \\
\text { percentage (\%) }\end{array}$} & 1. Test & & 6.51 & 10.66 & $8.34 \pm 1.4$ & \multirow{4}{*}{0.996} \\
\hline & 2. Test & \multirow[t]{2}{*}{14} & 6.59 & 10.74 & $8.34 \pm 1.4$ & \\
\hline & 3. Test & & 6.51 & 10.46 & $8.30 \pm 1.4$ & \\
\hline & Total & 42 & 6.51 & 10.74 & $8.33 \pm 1.3$ & \\
\hline
\end{tabular}

Notes. $p<0.05$; mmhg. is the abbreviation of millimetre(s) of mercury.

Table 3

Comparison of the Biometric Values of the Tekirova Belediyespor Football Team Between the Tests

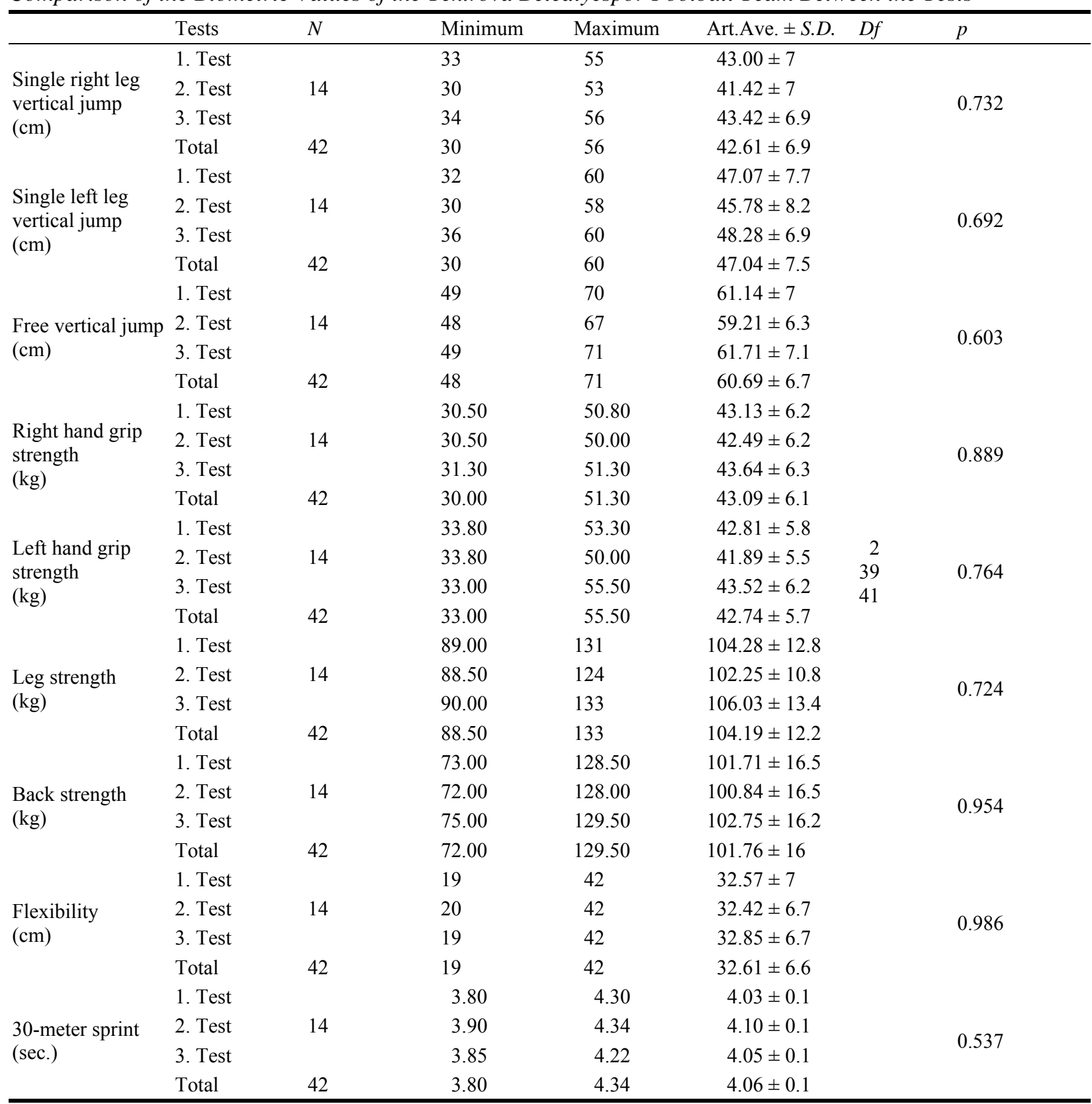

Notes. $p<0.05$; sec. is the abbreviation of second. 
Table 4

Comparison of the Physiological Values of the Tekirova Belediyespor Football Team Between the Tests

\begin{tabular}{|c|c|c|c|c|c|c|c|}
\hline & Tests & $N$ & Minimum & Maximum & Art.Ave. \pm S.D. & $D f$ & $p$ \\
\hline \multirow{4}{*}{$\begin{array}{l}\text { Systolic blood } \\
\text { pressure } \\
\text { (mmhg.) }\end{array}$} & 1. Test & \multirow{3}{*}{14} & 11.2 & 12.2 & $11.79 \pm 0.2$ & & \multirow{4}{*}{0.762} \\
\hline & 2. Test & & 11.3 & 12.3 & $11.79 \pm 0.3$ & & \\
\hline & 3. Test & & 11.5 & 12.3 & $11.86 \pm 0.2$ & & \\
\hline & Total & 42 & 11.2 & 12.3 & $11.81 \pm 0.2$ & & \\
\hline \multirow{4}{*}{$\begin{array}{l}\text { Diastolic blood } \\
\text { pressure } \\
\text { (mmhg.) }\end{array}$} & 1. Test & & 5.9 & 7.8 & $6.87 \pm 0.5$ & & \multirow{4}{*}{0.991} \\
\hline & 2. Test & 14 & 6.3 & 7.6 & $6.87 \pm 0.4$ & & \\
\hline & 3. Test & & 6.4 & 7.8 & $6.90 \pm 0.4$ & 2 & \\
\hline & Total & 42 & 5.9 & 7.8 & $6.88 \pm 0.4$ & 39 & \\
\hline \multirow{4}{*}{$\begin{array}{l}\text { Resting heartbeat } \\
/ \text { min. }\end{array}$} & 1. Test & & 61 & 68 & $63.71 \pm 2.2$ & 41 & \multirow{4}{*}{0.577} \\
\hline & 2. Test & 14 & 61 & 68 & $63.78 \pm 2.3$ & & \\
\hline & 3. Test & & 61 & 68 & $64.50 \pm 1.9$ & & \\
\hline & Total & 42 & 61 & 68 & $64.00 \pm 2.1$ & & \\
\hline \multirow{4}{*}{$\begin{array}{l}\text { Anaerobic } \\
\text { strength } \\
\text { (kg-m/sec.) }\end{array}$} & 1. Test & & 105.36 & 167.93 & $134.54 \pm 16$ & & \multirow{4}{*}{0.901} \\
\hline & 2. Test & 14 & 104.28 & 164.18 & $132.48 \pm 16$ & & \\
\hline & 3. Test & & 105.36 & 167.93 & $135.18 \pm 15$ & & \\
\hline & Total & 42 & 104.28 & 167.93 & $134.00 \pm 15.9$ & & \\
\hline
\end{tabular}

Notes. $p<0.05 ; \mathrm{kg}-\mathrm{m} / \mathrm{sec}$. is the abbreviation of kilogram-meter/second.

Table 5

Tekirova Belediyespor Versu (vs.) Sandıklı Spor (2-0) (20th Week). Game Analysis Through Tally Method

\begin{tabular}{|c|c|c|c|c|c|c|c|c|c|c|c|c|c|c|}
\hline & H.D. & Y.O. & İ.Ş. & G.K. & İ.Ö. & M.Ö. & G.A. & O.T. & C.Ç. & M.K. & U.K. & K.A. & O.K. & S.Y. \\
\hline Ball conceding & & 1 & 3 & 2 & 7 & & 9 & 5 & 2 & 1 & 9 & 3 & & 1 \\
\hline Ball stealing & & 3 & 9 & 1 & & 4 & 1 & 7 & 1 & 2 & 1 & 2 & 3 & \\
\hline Misplaced pass & 6 & 14 & 12 & 8 & 9 & 6 & 8 & 10 & 12 & 7 & 13 & 2 & 5 & 3 \\
\hline Successful pass & 12 & 43 & 47 & 38 & 28 & 13 & 25 & 35 & 29 & 31 & 37 & 5 & 11 & 5 \\
\hline Successful shot & & & 1 & 1 & & & & & & & & & & \\
\hline Unsuccessful shot & & 1 & 2 & 3 & 1 & 2 & 5 & 1 & & & 2 & 1 & 1 & \\
\hline Dribbling & & 3 & 2 & 7 & 2 & 9 & 2 & 3 & 1 & & 2 & 2 & & \\
\hline Right footswing (successful) & & 3 & 1 & & & & & & & & 1 & & & \\
\hline Right footswing (unsuccessful) & & 7 & & 3 & & 1 & & & & & 1 & 2 & 1 & \\
\hline Left foot swing (successful) & & & & & & & & 1 & & & & & 1 & \\
\hline Left foot swing (unsuccessful) & & 1 & & & & 1 & & 2 & & & & & 3 & \\
\hline Ball rejecting & & 4 & 6 & 1 & 1 & 2 & 2 & & 5 & 7 & 2 & 1 & 4 & 2 \\
\hline Clear the ball (successful) & & 3 & 5 & & 1 & & & 1 & 2 & 4 & & & & 1 \\
\hline Clear the ball (unsuccessful) & & 1 & 3 & 1 & & & & 3 & 4 & 1 & & & 1 & 3 \\
\hline Successful press & & 5 & 5 & 1 & & 4 & & 1 & & & 3 & & & \\
\hline Unsuccessful press & & 3 & 2 & & 2 & & 3 & & & & 4 & & & \\
\hline Successful fake & & 1 & 4 & 6 & 2 & 3 & 1 & 2 & 2 & & 12 & 1 & 2 & \\
\hline Unsuccessful fake & & 1 & 2 & 2 & 4 & 2 & 2 & 1 & & & 3 & & & \\
\hline Successful movement score & 32 & 131 & 161 & 113 & 73 & 74 & 67 & 103 & 84 & 93 & 116 & 27 & 47 & 23 \\
\hline Unsuccessful movement score & 18 & 58 & 50 & 40 & 49 & 27 & 57 & 46 & 41 & 23 & 66 & 20 & 25 & 19 \\
\hline Performance score & 14 & 73 & 111 & 73 & 24 & 47 & 10 & 57 & 43 & 70 & 50 & 7 & 22 & 4 \\
\hline
\end{tabular}


Table 6

Tekirova Belediyespor vs. Bergama Spor (1-2) (26th Week). Game Analysis by Tally

\begin{tabular}{|c|c|c|c|c|c|c|c|c|c|c|c|c|c|c|}
\hline & H.D. & Y.O. & İ.Ş. & G.K. & İ.Ö. & M.Ö. & G.A. & O.T. & C.Ç. & M.K. & U.K. & K.A. & O.K. & S.Y. \\
\hline Ball conceding & & 3 & 4 & 3 & 5 & & 4 & 6 & 3 & 1 & 9 & 3 & & 2 \\
\hline Ball stealing & & 2 & 5 & 1 & & 3 & 2 & 4 & 1 & 2 & 1 & 1 & 2 & \\
\hline Misplaced pass & 7 & 14 & 17 & 9 & 11 & 6 & 8 & 11 & 13 & 7 & 14 & 2 & 6 & 2 \\
\hline Successful pass & 8 & 28 & 30 & 20 & 24 & 12 & 22 & 29 & 24 & 31 & 28 & 4 & 9 & 4 \\
\hline Successful shot & & & & & & & 1 & & & & & & & \\
\hline Unsuccessful shot & & 1 & 4 & 3 & 1 & 3 & 4 & 1 & & & 3 & 1 & 1 & \\
\hline Dribbling & & 2 & 2 & 7 & 2 & 9 & 2 & 3 & 1 & & 2 & 2 & & \\
\hline Right foot swing (successful) & & 1 & 1 & & & & & & & & 1 & & & \\
\hline Right foot swing (unsuccessful) & & 8 & 2 & 4 & & 1 & & 1 & & & 1 & 2 & 1 & \\
\hline Left foot swing (successful) & & & & & & & & 1 & & & & & 1 & \\
\hline Left foot swing (unsuccessful) & & 1 & & & & 1 & 2 & 2 & & & & & 3 & \\
\hline Ball rejecting & & 5 & 4 & 1 & 1 & 3 & 2 & & 5 & 8 & 2 & 3 & 3 & 2 \\
\hline Clear the ball (successful) & & 3 & 4 & & 1 & & & 1 & 2 & 3 & & & & 1 \\
\hline Clear the ball (unsuccessful) & & 1 & 4 & 2 & & & & 4 & 3 & 1 & & & 1 & 2 \\
\hline Successful press & & 2 & 3 & 1 & 1 & 3 & & 1 & & & 2 & & & \\
\hline Unsuccessful press & & 5 & 2 & & 2 & & 3 & & & & 5 & & & \\
\hline Successful fake & & & 3 & 5 & 1 & 2 & 1 & 2 & 3 & & 9 & 2 & 2 & \\
\hline Unsuccessful fake & & & 5 & 3 & 5 & 2 & 3 & 2 & & & 5 & 4 & & \\
\hline Successful movement score & 25 & 88 & 106 & 74 & 64 & 68 & 64 & 84 & 76 & 94 & 93 & 30 & 41 & 20 \\
\hline Unsuccessful movement score & 21 & 83 & 77 & 50 & 51 & 29 & 50 & 54 & 43 & 23 & 76 & 27 & 27 & 18 \\
\hline Performance score & 4 & 21 & 29 & 24 & 13 & 39 & 14 & 30 & 33 & 71 & 17 & 3 & 14 & 2 \\
\hline
\end{tabular}

Table 7

Tekirova Belediyespor vs. Anadolu Üsküdarspor (3-0) (33rd Week). Game Analysis by Tally

\begin{tabular}{|c|c|c|c|c|c|c|c|c|c|c|c|c|c|c|}
\hline & H.D. & Y.O.A & İ.Ş. & G.K. & M.Ö. & G.A. & M.A. & O.T. & S.Y. & U.K. & D.A. & O.K. & A.E.A. & M.A.D. \\
\hline Ball conceding & & 3 & 4 & 2 & 2 & 9 & 2 & 5 & 2 & 9 & 1 & 1 & 3 & 3 \\
\hline Ball Stealing & & 7 & 10 & 2 & 3 & 1 & 4 & 7 & 8 & 1 & 3 & 2 & 3 & 3 \\
\hline Misplaced pass & 3 & 12 & 14 & 8 & 10 & 9 & 15 & 10 & 2 & 13 & 6 & 1 & 9 & 14 \\
\hline Successful pass & 13 & 35 & 39 & 20 & 28 & 24 & 32 & 30 & 10 & 31 & 16 & 8 & 22 & 23 \\
\hline Successful shot & & & & 3 & & & & & & & & & & \\
\hline Unsuccessful shot & & 1 & 2 & & 2 & 7 & & 1 & & 2 & & & & 2 \\
\hline Dribbling & & 4 & 3 & 7 & 9 & 5 & 5 & 3 & & 2 & 2 & & 4 & 3 \\
\hline Right foot cross (successful) & & 4 & 1 & 5 & & & & & & 4 & & & 5 & 5 \\
\hline Right foot cross (unsuccessful) & & 7 & 2 & 1 & 1 & & 1 & & & 1 & & & 1 & 2 \\
\hline Left foot cross (successful) & & & & & 4 & & 4 & 1 & & & & 2 & & \\
\hline Left foot cross (unsuccessful) & & 1 & & & 1 & & 1 & 2 & & & & 1 & & \\
\hline Ball rejecting & & 6 & 9 & 3 & 3 & 2 & 5 & 3 & 6 & 2 & 5 & 1 & 3 & 4 \\
\hline Clear the ball (successful) & & 4 & 6 & 2 & 1 & & 2 & 3 & 4 & & 6 & & 1 & 3 \\
\hline Clear the ball (unsuccessful) & & 1 & 3 & 1 & 1 & & & 1 & 2 & & 2 & & 1 & 1 \\
\hline Successful press & & 6 & 7 & 2 & 1 & 3 & 3 & 2 & 3 & 3 & 2 & & 2 & 3 \\
\hline unsuccessful press & & 3 & 2 & & & 3 & & & 1 & 4 & 1 & & 1 & 2 \\
\hline
\end{tabular}


(Table 7 to be continued)

\begin{tabular}{|c|c|c|c|c|c|c|c|c|c|c|c|c|c|c|}
\hline Successful fake & & 3 & 5 & 6 & 4 & 3 & 4 & 2 & & 12 & & 3 & 2 & 5 \\
\hline Unsuccessful fake & & & 2 & 2 & 2 & 1 & & 1 & & 3 & & 1 & 3 & 2 \\
\hline Successful movement score & 35 & 144 & 151 & 101 & 104 & 80 & 120 & 104 & 67 & 113 & 72 & 37 & 85 & 100 \\
\hline Unsuccessful movement score 1 & 13 & 56 & 60 & 31 & 39 & 61 & 40 & 42 & 18 & 66 & 24 & 12 & 29 & 53 \\
\hline Performance score & 22 & 88 & 91 & 70 & 65 & 19 & 80 & 62 & 49 & 47 & 48 & 25 & 56 & 47 \\
\hline
\end{tabular}

Notes. Each movement — whether successful or not—was multiplied by 2. If a player did not perform any of the above movements in the game, it was accepted as 1 point. Finally, the total of negative points was subtracted from the total of positive points, which yielded the performance score.

Table 8

Tekirova Belediyespor vs. Sandıklı Spor (2-0) (20th Week). Correlation Test Between the Performance Score and Some Biomotoric Qualities

\begin{tabular}{lllllllllll}
\hline $\begin{array}{l}\text { Free } \\
\text { vertical } \\
\text { jump }\end{array}$ & $\begin{array}{l}\text { Right foot } \\
\text { jump }\end{array}$ & $\begin{array}{l}\text { Left foot } \\
\text { jump }\end{array}$ & $\begin{array}{l}\text { Right hand Left hand } \\
\text { grip }\end{array}$ & grip & Back force Leg force & Flexibility & $\begin{array}{l}\text { 30-meter } \\
\text { sprint }\end{array}$ \\
\hline Performance score & $r=0.094$ & $r=0.055$ & $r=0.236$ & $r=0.11$ & $r=0.252$ & $r=0.039$ & $r=0.399$ & $r=0.094$ & $r=0.097$ \\
& $p=0.375$ & $p=0.426$ & $p=0.208$ & $p=0.486$ & $p=0.192$ & $p=0.447$ & $p=0.079$ & $p=0.375$ & $p=0.371$ \\
\hline
\end{tabular}

Notes. $-1<r<1 ; p<0.05$.

Table 9

Tekirova Belediyespor vs. Sandıklı Spor (2-0) (20th Week). Correlation Test Between the Performance Score and Some Physiological Test Values

\begin{tabular}{lllll}
\hline & Anaerobic power & Systolic blood pressure & $\begin{array}{l}\text { Diastolic blood } \\
\text { pressure }\end{array}$ & Pulse at rest \\
\hline Performance score & $r=0.347$ & $r=0.020$ & $r=0.029$ & $r=0.54$ \\
& $p=0.112$ & $p=0.902$ & $p=0.855$ & $p=0.104$ \\
\hline
\end{tabular}

Notes. $-1<r<1 ; p<0.05$.

Table 10

Tekirova Belediyespor-Bergamaspor (1-2) (26th Week). Correlation Test Between the Performance Score and Some Biomotoric Qualities

\begin{tabular}{llllllllll}
\hline $\begin{array}{l}\text { Free } \\
\text { vertical } \\
\text { jump }\end{array}$ & $\begin{array}{l}\text { Right foot } \\
\text { jump }\end{array}$ & $\begin{array}{l}\text { Left foot } \\
\text { jump }\end{array}$ & $\begin{array}{l}\text { Right hand } \\
\text { grip }\end{array}$ & $\begin{array}{l}\text { Left food } \\
\text { grip }\end{array}$ & Back force Leg force & Flexibility & $\begin{array}{l}\text { 30-meter } \\
\text { speed }\end{array}$ \\
\hline \multirow{2}{*}{ Performance score } & $r=0.250$ & $r=0.272$ & $r=0.255$ & $r=0.171$ & $r=0.129$ & $r=0.081$ & $r=0.223$ & $r=0.056$ & $r=0.353$ \\
& $p=0.388$ & $p=0.345$ & $p=0.378$ & $p=0.560$ & $p=0.659$ & $p=0.784$ & $p=0.443$ & $p=0.847$ & $p=0.216$ \\
\hline
\end{tabular}

Notes. $-1<r<1 ; p<0.05$.

Table 11

Tekirova Belediyespor vs. Bergamaspor (1-2) (26th Week). Correlation Test Between the Performance Score and Some Physiological Test Values

\begin{tabular}{|c|c|c|c|c|}
\hline & Anaerobic power & Systolic blood pressure & $\begin{array}{l}\text { Diastolic blood } \\
\text { pressure }\end{array}$ & Pulse at rest \\
\hline \multirow{2}{*}{ Performance score } & $r=0.091$ & $r=0.246$ & $r=0.312$ & $r=0.066$ \\
\hline & $p=0.757$ & $p=0.396$ & $p=0.276$ & $p=0.822$ \\
\hline
\end{tabular}

Notes. $-1<r<1 ; p<0.05$. 
Table 12

Tekirova Belediyespor vs. Anadolu Üsküdarspor (3-0) (33th Week). Correlation Test Between the Performance Score and Some Biomotoric Qualities

\begin{tabular}{llllllllll}
\hline & $\begin{array}{l}\text { Free } \\
\text { vertical } \\
\text { jump }\end{array}$ & $\begin{array}{l}\text { Right foot } \\
\text { jump }\end{array}$ & $\begin{array}{l}\text { Left foot } \\
\text { jump }\end{array}$ & $\begin{array}{l}\text { Right hand Left hand } \\
\text { grip }\end{array}$ & grip & Back force Leg force & Flexibility & $\begin{array}{l}\text { 30-meter } \\
\text { sprint }\end{array}$ \\
\hline Performance & $r=0.144$ & $r=0.282$ & $r=0.179$ & $r=0.093$ & $r=0.314$ & $r=0.014$ & $r=0.193$ & $r=0.186$ & $r=0.098$ \\
score & $p=0.624$ & $p=0.385$ & $p=0.541$ & $p=0.752$ & $p=0.275$ & $p=0.962$ & $p=0.509$ & $p=0.523$ & $p=0.738$ \\
\hline
\end{tabular}

Notes. $-1<r<1 ; p<0.05$.

Table 13

Tekirova Belediyespor vs. Anadolu Üsküdarspor (3-0) (33th Week). Correlation Test Between the Performance Score and Some Physiological Test Values

\begin{tabular}{lllll}
\hline & Anaerobic power & $\begin{array}{l}\text { Systolic } \\
\text { blood pressure }\end{array}$ & $\begin{array}{l}\text { Diastolic } \\
\text { blood pressure }\end{array}$ & Pulse at rest \\
\hline Performance score & $r=0.338$ & $r=0.249$ & $r=0.601$ & $r=0.223$ \\
& $p=0.237$ & $p=0.389$ & $p=0.220$ & $p=0.441$ \\
\hline
\end{tabular}

Notes. $-1<r<1 ; p<0.05$.

When the literature is reviewed, it is noticed that there is a typical body structure, which is common among football players. An average height of $180 \mathrm{~cm}$, body weight proportioned with the height, namely, a mesomorph somatotype and a body fat percentage of 7 to 12. Günay and Yüce (2001) conducted a study on the footballers of Tepecik Belediyespor and Bakırköy Belediyespor, the third league Turkish football teams, and found the following values: For Tepecik Belediyespor players, the average height was $179.6 \pm 4.97 \mathrm{~cm}$, the average weight was $74.85 \pm 4.48 \mathrm{~kg}$, and the average age was $25.95 \pm 4.98$ years. For Bakırköy Belediyespor, the average height was $179.2 \pm 4.52 \mathrm{~cm}$, the average weight was $78.84 \pm 3.96 \mathrm{~kg}$, and the average age was 22.74 \pm 4.22 years. It is seen that the average weights of the teams were $74.85 \pm 4.48 \mathrm{~kg}$ for Tepecik and $78.84 \pm 3.96$ $\mathrm{kg}$ for Bakırköyspor (Kesler, Kaya, Ateş, \& Şahin, 2003). In the present study, which was conducted on Tekirova Belediyespor, which is a third league football team, it was determined that the average age, height, and weight of the 14 football players, who played in the games, in the 20th, 26th, and 33rd weeks were $24.28 \pm$ 2.63 years, $180 \pm 6.48 \mathrm{~cm}$, and $77.78 \pm 7.31 \mathrm{~kg}$, respectively (see Table 1).

Müniroğlu, Koz, Atıl, Erongun, and Bulca (2000) found the body fat percentage as $6.43 \pm 1.67$ for Turkish super league football players. Strudwick, Reilly, and Doran (2002) conducted a study on Welsh and English football players in 2002. They compared the footballers in terms of anthropometric differences for the whole of a season and determined a body fat of $11.2 \%$. The football players $(N=24)$ from Marmarisspor, a third division football team in Turkey, voluntarily participated in a previous study. In the study, the body fat percentage was determined as $9.01 \pm 1.22$ for the pre-season and $8.18 \pm 0.10$ for the mid-season (Saygin, 2001). As for our study, the body fat percentages for the first, second, and third tests were found as $8.34 \pm 1.4,8.34 \pm 1.4$, and $8.30 \pm 1.4$, respectively (see Table 2 ).

In the same study, leg force values for the pre- and post- test were found as $124.35 \pm 8.79 \mathrm{~kg}$ and $138.42 \pm$ $10.41 \mathrm{~kg}$, respectively (Saygin, 2001). In another study conducted on super league football players in Turkey, the leg force was found to be $138.74 \pm 19.08 \mathrm{~kg}$ (Aslan, İnan, \& Akalan, 2010). In our study, the average leg force for the first, second, and third tests were found to be $104.28 \pm 12.8 \mathrm{~kg}, 102.25 \pm 10.8 \mathrm{~kg}$, and $106.03 \pm$ $13.4 \mathrm{~kg}$ (see Table 3). 
In a study conducted on professional players, the right hand grip force was found as $48.78 \pm 39 \mathrm{~kg}$ and the left hand grip force was found as $47.24 \pm 3.8 \mathrm{~kg}$ (Çebi, 1999). In our study, the average right hand grip force was found $43.13 \pm 6.2 \mathrm{~kg}$ for the first test, $42.49 \pm 6.2 \mathrm{~kg}$ for the second test, and $43.64 \pm 6.3 \mathrm{~kg}$ for the third test, and the average left hand grip force values were found as $42.81 \pm 5.8 \mathrm{~kg}, 41.89 \pm 5.5 \mathrm{~kg}$, and $43.52 \pm 6.52$, respectively (see Table 3).

Ostojic (2004) determined the vertical jump height of the football players as $49.9 \pm 7.5 \mathrm{~cm}$. Cerrah, Polat, and Ertan (2011) conducted a study on super amateur football players in 2011 and found that the vertical jump values varied according to the positions of the players in a football pitch (Goal: $34.7 \pm 4.5$; Defense: $34.6 \pm 4.4$; Midfield: $34.8 \pm 4.5$; Forward: $36.1 \pm 6.2$ ) and active (Goal: $37.6 \pm 4.1$; Defense: $36.7 \pm 4.3$; Midfield: $37.2 \pm 5$; Forward: $38.8 \pm 5.2$ ). In a study conducted on 24 male football players, the average vertical jump test score was found as $55 \pm 5.7 \mathrm{~cm}$ (Kamar et al., 2003). In the present study, the average free vertical jump values were $61.14 \pm 7 \mathrm{~cm}, 59.21 \pm 6.3 \mathrm{~cm}$, and $61.71 \pm 7.1 \mathrm{~cm}$, respectively (for the first, second, and third tests). The right foot vertical jump values were $43 \pm 7 \mathrm{~cm}, 41.42 \pm 7 \mathrm{~cm}$, and $43.42 \pm 6.9 \mathrm{~cm}$, respectively. The left foot vertical jump values were found as $47.07 \pm 7.7 \mathrm{~cm}, 45.78 \pm 8.2 \mathrm{~cm}$, and $48.28 \pm 6.9 \mathrm{~cm}$, respectively (see Table 3).

In a study conducted on Turkish super league football players, the average flexibility was found to be $30.96 \pm 6.66$ (Aslan, İnan, \& Akalan, 2010). Müniroğlu et al. (2000) found an average flexibility value of 31.57 $\pm 5.78 \mathrm{~cm}$. In the present study, the average flexibility values were found as $32.57 \pm 7 \mathrm{~cm}, 32.42 \pm 6.7 \mathrm{~cm}$, and $32.85 \pm 6.7 \mathrm{~cm}$, respectively (see Table 3 ).

In a study conducted on volunteer football players $(N=24)$ from Marmarisspor, a third league football team in Turkey, the average 30-meter sprint rates of the footballers were found to be $4.14 \pm 0.1 \mathrm{sec}$. for pre-training and $4.06 \pm 0.127 \mathrm{sec}$. for post-training during the preparation season (Saygın, 2001). In another study, the average 30-meter sprint rate was found to be $4.28 \pm 0.16 \mathrm{sec}$. (Kizilet et al., 2004). Whereas in the present study, the average 30 -meter sprint test rates were found to be $4.03 \pm 0.1 \mathrm{sec}$., $4.10 \pm 0.1 \mathrm{sec}$., and $4.05 \pm$ $0.1 \mathrm{sec}$., respectively (see Table 3 ).

Heart rate at rest was found to be $72.28 \pm 9.39$ beats $/ \mathrm{min}$. for the pre-test and $62.56 \pm 9.08$ beats $/ \mathrm{min}$. for the post-test, which means that there is a statistically significant difference $(p<0.05)$, which may suggest that the players reacted positively to trainings, and this situation caused that the heart worked more economically (Erkmen, Kaplan, \& Taşkın, 2005). In the present study, the average heart rates at rest were found $63.71 \pm 2.2$ beats/min., $63.78 \pm 2.3$ beats $/ \mathrm{min}$., and $64.50 \pm 1.9$ beats $/ \mathrm{min}$., respectively (see Table 4).

Systolic blood pressure values were $110.00 \pm 11.88 \mathrm{mmhg}$. for the pre-test and $110.56 \pm 11.62 \mathrm{mmhg}$. for the post-test. Diastolic blood pressures were found as $73.89 \pm 7.78 \mathrm{mmhg}$. and $74.44 \pm 5.11 \mathrm{mmhg}$., respectively, and no significant difference was found (Erkmen, Kaplan, \& Taşkın, 2005). In our study, systolic and diastolic blood pressures were found as $11.79 \pm 0.2$ and $6.87 \pm 0.5 \mathrm{mmhg}$. for the first test; $11.79 \pm 0.3$ and $6.87 \pm 0.4 \mathrm{mmhg}$. for the second test, and $11.86 \pm 0.2$ and $6.90 \pm 0.4 \mathrm{mmhg}$. for the third test, respectively (see Table 4).

In a study conducted on volunteer football players $(N=24)$ from Marmarisspor, a third league football team in Turkey, while the anaerobic power was $101.14 \pm 5.98 \mathrm{~kg}-\mathrm{m} / \mathrm{sec}$. at the beginning of the preparation season, it was determined $106.05 \pm 4.83 \mathrm{~kg}-\mathrm{m} / \mathrm{sec}$. after the preparation season, and thus, a significant relationship $(p<0.01)$ was found. In the present study, the average anaerobic power was found as $134.54 \pm 16$ $\mathrm{kg}-\mathrm{m} / \mathrm{sec}$. for the first test, $132.48 \pm 16 \mathrm{~kg}-\mathrm{m} / \mathrm{sec}$. for the second test, and $135.18 \pm 15 \mathrm{~kg}-\mathrm{m} / \mathrm{sec}$. for the third test (see Table 4). 
Zıvalıoğlu (1997) analyzed the techniques in the games between Trabzonspor and Bursaspor, Trabzonspor and Ankaragücü, Trabzonspor and Kocaelispor, and Trabzonspor and Beşiktaş. In this study, a statistically significant difference was found in terms of ball gaining within the game $(p<0.05)$. In our study, a statistically significant difference could not be determined between the values of the combined performance analysis values and actual game performance levels $(p>0.05)$. The training and game performances of the players correspond to each other. In the literature review, no studies were observed on game performance levels that depended on combined performance analysis (see Tables 5-13).

\section{Conclusion}

The present study was designed for the purpose of finding a statistically significant result between the performance of football players during games and their training performances. No significant relations were determined in the analyses. In case the trainers have problems in forming the team players, they may have an idea on which player will contribute more during the game in a combined performance analysis, which will be made two days before the actual game. While new approaches and collective solutions develop in a fast pace in today's football world, new methods with more numerical importance and concrete results must be produced together with the contributions of the positive science to sports. When the duration of the study is considered, it is possible to claim that as the number of the games that are evaluated increases, it becomes more probable to reveal the relation between the game performance and the combined performance analysis in a statistical manner.

\section{References}

Aslan, C. S., İnan, T., \& Akalan, C. (2010). Comparison of some physical and physiological properties of physical education and sports high school students and a professional footbal team. Journal of New World Sciences Academy Sports Sciences, 5(1), 47-58.

Cerrah, A., Polat, C., \& Ertan, H. (2011). Examining some physical and technical parameters of the footballers of super amateur league according to their positions. Nigde University Journal of Physical Education and Sport Sciences, 5(1), 1-6.

Carling, C., Williams, A. M., \& Reily, T. (2006). Handbook of soccer match analysis: A systematic approach to improving performance. Journal of Sports Science and Medicine, 5(1), 171.

Çebi, M. (1999). Comparison of physiological parameters in amateur and professional sportsmen (Postgraduate thesis, Physial Education and Sport Department, Institute of Healthcare Sciences, Mayis University, Samsun).

Duyul, M. (2005). Comparison of the effects of some motoric and anthropometric properties of handball, volleyball, and football university teams on success (Postgraduate thesis, Healthcare Sciences Institute, May1s University, Samsun).

Erkmen, N., Kaplan, T., \& Taşkın, H. (2005). Determining and comparing the physical and physiological parameters of professional footballers in preseason. Spormetre Journal of Physical Education and Sport Sciences, 3(4), 137-144.

Günay, M., \& Yüce, A. (2001). Scientific basis of football training (2nd ed.). Ankara: Gazi Bookstore.

Kamar, A., Güngördü, O., Yüceyılmaz, B., Ataman, H., Çavuşoğlu, B., \& Şahin, M. (2003). Scores of football players in 35-meter maximal anaerobic sprint and vertical jump and standing long-jump. Istanbul University Journal of Sport Science, 11(3), 147-150.

Kesler, A., Kaya, B., Ateş, O., \& Şahin, M. (2003). The effects of training on maximal oxygen capacities of professional footballers. I. U. Journal of Sports Sciences, 11(3), 80-83.

Kızılet, A., Erdem, K., Karagözoğlu, C., Topsakal, N., \& Çalışkan, E. (2004). Assessment of some physical and motoric properties in terms of position in footballers. Gazi Journal of Physical Education and Sport Sciences, 9(3), 67-78.

Kilınç, F. (2008). An Intensive combined training program modulates physical, physiological, biomotoric, and technical parameters in basketball player women. Journal of Strength and Conditioning Research, 22(6), 1769-1778.

Keyserling, W. M., Herrin, G. D., \& Chaffin, D. B. (1978). An analysis of selected work musle strength. Proceedig of Human Factors Society 22nd Annual Meeting, Detroit, USA. 
Müniroğlu, S., Koz, M., Atıl, M., Erongun, D., \& Bulca, Y. S. (2000). Examining the physical and physiological properties of a football team in Turkey professional first league before and after the season. First Gazi Physical Education and Sports Sciences Congress. Book of Movement and Training Knowledge (Vol. 1). Ankara, Turkey: Sim Publishing House.

Ostojic, S. M. (2004). "Elite and nonelite soccer players: Preseasonal physical and physiological characteristics" research. Sports Medicine, 12 (2), 143-150.

Saygın, Ö. (2001). The effect of preseasonal training programs on some physical and physiological properties of professional footballers. Journal of Physical Education and Sport, 1(3), 102-107.

Strudwick, A., Reilly, T., \& Doran, D. (2002). Antropometric and fitness profiles of elite players in two football codes. The Journal of Sports Medicine and Physical Fitness, 42(2), 239-242.

Zıvalıoglu, H. D. (1997). Analysis of the technical movements of trabzonspor football team in the field (Postgraduate thesis, Healthcare Sciences Institute, Karadeniz Teknik University, Trabzon). 